\title{
Personally Identifiable Information: Identifying unprotected PII using file-indexing search tools and quantitative analysis
}

\author{
Brandis W. Matthews \\ Department of Computer Science \\ North Carolina A\&T State University \\ Greensboro, NC \\ brandis.matthews@yahoo.com
}

\author{
Dr. Albert Esterline \\ Department of Computer Science \\ North Carolina A\&T State University \\ Greensboro, NC \\ esterlin@ncat.edu
}

\begin{abstract}
This paper reports on a project in which employees at Federal agencies are surveyed regarding their knowledge of security policies in place in their offices and their knowledge of PII (Personally Identifiable Information) stored on their systems.
\end{abstract}

\section{INTRODUCTION}

Privacy and confidentiality of sensitive data is one of the most critical concerns of private citizens and information technology managers. Many corporations and Federal agencies, when faced with the loss of data, immediately call their IT department for help in recovering the data. The truth is that most organizations do not know how much data has been lost, the sensitivity of the data, and the potential impact of the loss if leaked to the public. They also cannot say where exactly the data is stored. For example, in January of 2009, the U.S. Consulate in Jerusalem reported that hundreds of files containing Social Security numbers, bank account numbers, and other sensitive data for an unknown number of people were found in a filing cabinet purchased from the Consulate at an auction in Jerusalem [1]. Most electronic data breaches occur because information is stored on portable hardware or public drives instead of local drives that are only accessible by individual employees. The National Guard, IRS, and Department of Human Services have all reported breaches of data that occurred through hacking or hardware being stolen [1].

Personally Identifiable Information (PII) is defined by the Office of Management and Budget as information, such as Social Security number, date of birth, mother's maiden name, or biometric records, that can be used alone or combined to identify or suggest an individual's identity [2]. How is information classified on your system? Does this information contain PII? If leaked, what level of compromise will occur? These are the types of questions posed during an information technology audit when a data breach occurs within an organization. If asked these questions, would you be able to give a specific, detailed response? With an increase in online networking and information sharing, agencies must implement policies to prevent data breaches.

Knowing where PII is stored and who has access to it is critical. It is vital to clarify who is responsible for the information and who has privileged rights to access or change the information. All Federal agencies are responsible for implementing a policy identifying individuals authorized to access PII or sensitive information and outlining proper handling of PII by all employees [3]. The policy should also disclose the responsibilities of authorized individuals as well as the consequences for violating the policy [3]. Confidential, secret, or top secret information should not be accessible to the public under any circumstances. Defining roles and policies that cover activities from collecting the data to disposal of the data is an important part of maintaining secure access.

This paper addresses a project in which employees at Federal agencies are surveyed regarding their knowledge of security policies in place in their offices and their knowledge of PII stored on their systems. The latter is compared with a scan for PII to identify PII whose existence is unknown to the employee storing it. The scans and surveys together thus indicate the level at which employees are unaware of PII on their systems and the actual effectiveness of current policies. We also survey Information Security Systems Officers (ISSOs) to determine the extent to which they are aware of how PII is handled within their security boundaries and how their handling conforms to mandated policy. The eventual goal is to suggest improved policies and ways to promote them. 


\section{FILE-INDEXING SEARCH TOOLS}

To investigate the extent to which unprotected, sensitive data can be found even on secure network drives, free file indexing tools have been explored [4],[5]. Since this investigation deals with scanning for sensitive information within environments, it is important to have a program that will search local drives, search network drives, permit regular expression searches, and filter results based on file extensions including those used in Microsoft Office 1997-2007 (e.g., .xls; .pdf; .txt; .doc; .mdb; .docx; .xlsx; .mde; .wpd).

A regular expression, or pattern, is used to describe a set of strings [6]. In many file-indexing tools, regular expressions are used to search for specific string values within file systems. For this investigation, regular expressions include search strings for Social Security numbers and all major credit card numbers. Social Security Numbers are the most exposed PII in Federal organizations [7]. If a regular expression search returns a Social Security number accompanied by other PII such as a credit card number or date of birth, the results in the wrong hands can be devastating [8]. To search for Social Security numbers, the string is defined as a sequence of decimal digits and dashes. Credit card numbers are more sophisticated in the coding as each major credit card has a distinct pattern. Previous investigations of searching for PII using regular expressions have focused solely on personal computer systems and networks [7].

Several file-indexing tools considered for this investigation are Swish-e, Nessus, PowerGrep, Microsoft Indexing Service, MariusSoft's File Search, Agent Ransack, SnowBird, and Binary Fortress' Fileseek. Another tool, PowerShell, was used by Frank Simorjay to search for PII using regular expressions on his personal computer [7]. Although PowerShell is a useful tool, it requires more scripting and debugging than desirable. After testing the various tools, PowerGrep and Nessus were found to provide everything necessary for the investigation. PowerGrep and Nessus use regular expressions to search for specific string values within file systems. However, PowerGrep was found to be the best tool to retrieve sensitive material stored on Microsoft Windows systems and network drives. PowerGrep [9] is a grep tool designed to quickly search through large quantities of text and binary files. Two distinct advantages PowerGrep has over other file indexing services are the ability to search local and network drives and the ability to search using multiple regular expressions at the same time. Multiple regular expression searches allow one to focus on information that is particularly relevant [10].

Nessus [11], a scanning utility used by many government organizations, is used in addition to PowerGrep to scan for a subset of PII (e.g., Social Security number and credit card number). Nessus, a real-time vulnerability scanner, provides audit reports, asset profiling, sensitive data discovery, and vulnerability analysis of a system. Nessus does not require installation on the systems that will be scanned. Nessus can scan multiple client systems simultaneously using regular expression searches. It will return specific file names, locations, and the byte sizes from a remote Windows system. When the files to be scanned are stored on a network drive, PowerGrep is used since Nessus does not support scanning network drives.

\section{SURVEY AND DATA ANALYSIS}

PowerGrep scans return the PII found and the location of the file containing the PII. The scan results are compiled in a separate document and used as part of the statistical analysis. Used in conjunction with a written survey, an ISSO can use the scan results to better assess the potential for a breach of PII. In order to obtain a detailed response, the survey will be conducted by sampling two distinct populations. The first survey will be directed towards the employee of the system being scanned. An ISSO will complete the second survey. The employee survey questions concentrate on employee knowledge of PII and policies regarding sensitive information in their organization. The employee survey will allow employees at a Federal organization to give a written account of whether they have knowledge of PII on their systems, whether it is properly secured, and whether they know the local policies and procedures for securing PII. The survey opens by asking the employee where the policies in place for securing and storing sensitive information can be found. It then moves on to identifying workstations and servers that contain sensitive information. It also asks the employee to identify the directories and folders where the data can be found and who has access to this information. The results of the employee survey will show the number of employees who are unaware of PII on their system, the number of employees who know where PII and sensitive information are stored, and the number that are complying with current policies regarding PII.

The ISSO survey answers questions pertaining to who, in terms of role, is allowed to store and access PII at a specific location. For this survey, role is defined as the job function and responsibility of an employee (for example, HR personnel, secretary, or system administrator). One goal of this survey is to determine whether ISSOs at Federal organizations can identify all applicable locations containing PII. This survey also identifies whether data is encrypted in transit or while it is backed up. Some questions in the survey focus on policies in place for sensitive information. The results of the ISSO survey reveal whether the organization is encrypting PII during backups and in transit. The results will also show the extent to which ISSOs are aware of how PII is handled within their security boundaries.

Obtaining solid results from the scans and surveys is vital to a strong statistical analysis. The data analysis will analyze scans and surveys involving a subset of PII from a sample 
population. The analysis will show the extent to which PII exists on systems used by the organizations in question, the extent to which employees are unaware of PII on their systems, and the extent to which policies are known and being followed. Thus, the analysis can provide an estimate of the probability that any system has PII. If we multiply this by the number of systems in each set of scans, we will have the expected mean. The analysis can also provide the expected types of PII to be found, and the probability that PII would be found on other systems. The analysis will thus suggest the extent to which PII can be compromised in the organizations. The statistical analysis will hopefully give researchers an idea of how to better protect access to PII to reduce the number of compromises occurring annually.

\section{PRELIMINARY RESUltS}

Preliminary system scans revealed unprotected PII on employees' local and network drives. The surveys obtained so far show employees are aware of PII on their systems and know where it is located. From anecdotal evidence, however, we expect to find cases where the employees are not aware of the security policies and do not know that PII exists on their system. If the survey responses vary significantly in employee knowledge of PII and security policies from office to office, further research will be needed to identify whether the knowledge of security policies and presence of PII is a result of certain aspects of the office culture.

\section{CONCLUSION}

PII is any combination of personal data that can be used to identify you. The purpose of this investigation is to find the level at which employees and ISSOs are unaware of PII on their systems, the level at which employees and ISSOs follow current policies regarding PII. File-indexing search tools were used to perform a regular expression search for a subset of PII (viz., Social Security numbers and credit card numbers). The results from the scans and the survey completed by each Federal office will give organizations a clearer outlook on how they protect and secure PII. Each Federal organization is responsible for classifying its own data and making sure it is in compliance with FIPS 199 [12]. FIPS 199 is designed to develop standards for categorizing information and information systems. It defines three levels of impact upon an organization or individual given a breach of security. The impact of a loss of PII in any organization can be limited, serious, or catastrophic. Future work can involve additional surveying of the employees and ISSOs to investigate attitudes and experiences. For example, failure to follow policy consistently might relate to employees not considering security at their work place a serious issue. It might also relate to a lack of experience with security. Future work can also consist of using the file indexing tools in addition to PII as search criteria to investigate different ways online social networking site profiles can be breached [13].

\section{REFERENCES}

[1] "A Chronology of Data Breaches," Privacy Rights ClearingHouse, April 20, 2005. [Online] Available:

http://www.privacyrights.org/ar/ChronDataBreaches.htm [Accessed: Sept. 29, 2009]

[2] C. Johnson, "Safeguarding Against and Responding to the Breach of Personally Identifiable Information," Office of Management and Budget, May 22, 2007. [Online] Available:

http://www.whitehouse.gov/omb/memoranda/fy2007/m07-16.pdf. [Accessed: Sept. 17, 2009]

[3] E. McCallister, T. Grance, K. Scarfone, "Guide to Protecting the Confidentiality of Personally Identifiable Information (PII)," NIST Special Publication 800-122 (Draft). January 2009.

[4] WebAttack Inc, "SnapFiles - Software reviews, download freeware and free trial programs," 1997.[Online] Available: http://www.webattack.com. [Accessed: Nov. 12, 2009]

[5] A. Lee, "The Portable Freeware Collection - Latest entries," 2004. [Online] Available: http://www.portablefreeware.com. [Accessed: Nov. 12, 2009]

[6] J. Hopcroft, R. Motwani, J. D. Ullman, Introduction to Automata Theory, Languages, and Computation, $3^{\text {rd }}$ ed., Addison-Wesley, 2006.

[7] F. Simorjay. "Security Watch: Where Is My PII?"Microsoft TechNet Magazine. April 2008

[8] B. Bain and M. Mosquera, "FAA breach heightens cybersecurity concerns," Federal Computer Week. Feb. 23, 2009.

[9] Just Great Software Co. Ltd., "Windows Grep Software," November 22, 2002. [Online] Available: http://www.powergrep.com. [Accessed: Sept. 1, 2009]

[10] X. Shen, B. Tan, and C. Zhai, "Privacy Protection in Personalized Search," ACM SIGIR Forum, Vol. 41 No.1. June 2007.

[11] Tenable Network Security(R), "Nessus: Network Vulnerability Scanner," September 2002. [Online] Available: http://www.nessus.org/nessus/. [Accessed: Aug. 19, 2009]

[12] Computer Security Division, "Standards for Security Categorization of Federal Information and Information Systems," NIST:FIPS-PUB-199, February 2004

[13] B. Krishnamurthy and C. Wills, "On the Leakage of Personally Identifiable Information Via Online Social Networks," ACM Workshop on Online Social Networks, August 17, 2009. 